\title{
ON CANONICAL FORMS OF DIFFERENTIAL EQUATIONS*
}

\author{
BY V. G. GROVE
}

1. Introduction. The projective differential geometry of surfaces has been greatly enriched by the introduction of Fubini's projective normal into the theory. This projective normal however fails to exist for ruled surfaces. In this paper we propose a class of congruences covariantly related to the surface, each congruence being suitable as a substitute for Fubini's projective normal congruence. As an application we give an interesting class of congruences covariantly related to a scroll surface and conjugate to the surface.

If the asymptotic parameters are parametric, the four homogeneous coordinates $y^{(1)}, y^{(2)}, y^{(3)}, y^{(4)}$ of a general point on the surface $S_{y}$ are solutions of differential equations in the Wilczynski semicanonical form: $\dagger$

$$
\left\{\begin{array}{l}
y_{u u}+2 a y_{u}+2 b y_{v}+c y=0, \\
y_{v v}+2 a^{\prime} y_{u}+2 b^{\prime} y_{v}+c^{\prime} y=0 .
\end{array}\right.
$$

One of the integrability conditions of system (1) is

$$
a_{v}=b_{u}^{\prime} \text {. }
$$

If the transformations

$$
y=\lambda \bar{y}
$$

and

$$
\bar{u}=U(u), \quad \bar{v}=V(v)
$$

are performed on system (1), the new coefficients are respectively

$$
\left\{\begin{array}{lll}
\bar{a}=a+\frac{\lambda_{u}}{\lambda}, & \bar{b}=b, & \bar{c}=\frac{1}{\lambda}\left(\lambda_{u u}+2 a \lambda_{u}+2 b \lambda_{v}+c \lambda\right), \\
\bar{a}^{\prime}=a^{\prime}, & \bar{b}^{\prime}=b^{\prime}+\frac{\lambda_{v}}{\lambda}, & \bar{c}^{\prime}=\frac{1}{\lambda}\left(\lambda_{v v}+2 a^{\prime} \lambda_{u}+2 b^{\prime} \lambda_{v}+c^{\prime} \lambda\right),
\end{array}\right.
$$

* Presented to the Society, April 19, 1930.

$\dagger$ E. J. Wilczynski, First memoir, Transactions of this Society, vol. 8 (1907), pp. 233-260. 
and

$$
\left\{\begin{array}{c}
\bar{a}=\frac{1}{U_{u}}\left(a+\frac{1}{2} \frac{U_{u u}}{U_{u}}\right), \quad \bar{b}=\frac{V_{v}}{U_{u}^{2}} b, \quad \bar{c}=\frac{c}{U_{u}^{2}} \\
a^{\prime}=\frac{U_{u}}{V_{v}^{2}} a^{\prime}, \quad \bar{b}^{\prime}=\frac{1}{V_{v}}\left(b^{\prime}+\frac{1}{2} \frac{V_{v v}}{V_{v}}\right), \quad \bar{c}^{\prime}=\frac{c^{\prime}}{V_{v}^{2}} .
\end{array}\right.
$$

2. The Canonical Forms. Consider an invariant $R$ of the surface with the following properties:

(a) $R \neq 0$, (b) the transform $\bar{R}$ of $R$ by (4) is $\bar{R}=R /\left(U_{u} V_{v}\right)$.

Condition (2) implies the existence of a function $p$ such that

$$
p_{u}=a+h \frac{R_{u}}{R}, \quad p_{v}=b^{\prime}+h \frac{R_{v}}{R},
$$

where $h$ is a constant. The transforms $\bar{p}_{u}$ and $p_{v}$ of $p_{u}$ and $\bar{p}_{v}$ by means of (3) are

$$
\bar{p}_{u}=p_{u}+\frac{\lambda_{u}}{\lambda}, \quad \bar{p}_{v}=p_{v}+\frac{\lambda_{v}}{\lambda} .
$$

If we examine the transforms of $p_{u}$ and $p_{v}$ under (4), we observe that these functions will be invariant under that transformation if and only if $h=1 / 2$. Hence the points $r$ and $s$ defined by the formulas

$$
\begin{aligned}
r & =y_{u}+p_{u} y, & s & =y_{v}+p_{v} y, \\
p_{u} & =a+\frac{1}{2} \frac{R_{u}}{R}, & p_{v} & =b^{\prime}+\frac{1}{2} \frac{R_{v}}{R},
\end{aligned}
$$

are covariant points. Transformation (3) for $\lambda=e^{-p}$ transforms the expressions $r$ and $s$ into $y_{u}$ and $y_{v}$ respectively. With this choice of $\lambda$, system (1) assumes the following canonical form:

$$
y_{u u}=\theta_{u} y_{u}+\beta y_{v}+P y, \quad y_{v v}=\gamma y_{u}+\theta_{v} y_{v}+Q y,
$$

wherein

$$
\begin{aligned}
& \theta=\log R, \quad \beta=-2 b, \quad \gamma=-2 a^{\prime} \\
& P=-\Phi=-\left[c-a_{u}-a^{2}-2 b b^{\prime}-b \theta_{v}-\frac{1}{2} \theta_{u u}+\frac{1}{4} \theta_{u}^{2}\right] \\
& Q=-\Psi=-\left[c^{\prime}-b_{v}-b^{\prime 2}-2 a a^{\prime}-a^{\prime} \theta_{u}-\frac{1}{2} \theta_{v v}+\frac{1}{4} \theta_{v}^{2}\right]
\end{aligned}
$$

The quadratic form

\section{$2 R d u d v$}


is absolutely invariant under (3) and (4). If we compute the second covariant derivatives of $y$ with respect to the form (13), we find that the four functions $y$ satisfy the equations

$$
y_{11}=\beta y_{2}+P y, \quad y_{22}=\gamma y_{1}+Q y,
$$

wherein $y_{1}=y_{u}, y_{2}=y_{v}$.

The coordinates $y$, which satisfy (11) or (14), are similar to Fubini's normal coordinates. In fact if $R=a^{\prime} b$ the canonical form (14) becomes Fubini's canonical form and the invariants $\Phi$ and $\Psi$ become the invariants $\phi$ and $\psi$ derived by Lane.*

3. Geometric Considerations. We shall call the line joining $y$ to the covariant point $y_{u v}$ the $R$ conjugate line, and the line joining the covariant points $y_{u}, y_{v}$ the $R$ harmonic line. The congruences generated by these lines are respectively conjugate $\dagger$ and harmonic to the surface.

A geometric characterization of the $R$ conjugate line of course depends upon the particular invariant $R$. In terms of this characterization, geometric interpretations of the covariants $y_{u}$, $y_{v}$, and $y_{u v}$ and of the vanishing of the invariants $P$ and $Q$ parallel the work of Lane in the paper just cited.

The developables of the congruence of $R$ conjugate lines intersect $S_{y}$ in the integral curves of the differential equation

$$
\left(\Phi+2 b_{v}+2 b \theta_{v}\right) d u^{2}-\left(\Psi+2 a_{u}^{\prime}+2 a^{\prime} \theta_{u}\right) d v^{2}=0 .
$$

The developables of the congruence of $R$ harmonic lines correspond to the curves defined by the equation

$$
\Phi d u^{2}-\Psi d v^{2}=0 .
$$

Referred to the tetrahedron $y, y_{u}, y_{v}, y_{u v}$ of system (11) the equation of the quadric of Lie is

$$
x_{1} x_{4}-x_{2} x_{3}+\left(2 a^{\prime} b+\frac{1}{2} \theta_{u v}\right) x_{4}{ }^{2}=0 .
$$

The $R$ conjugate line intersects this quadric in $y$ and in the point defined by

* E. P. Lane, Wilczynski's and Fubini's canonical systems of differential equations, this Bulletin, vol. 32 (1926), p. 368.

$\dagger$ G. M. Green, Memoir on the general theory of surfaces and rectilinear congruences, Transactions of this Society, vol. 20 (1919), p. 99. 


$$
y_{u v}-\left(2 a^{\prime} b+\frac{1}{2} \theta_{u v}\right) y \text {. }
$$

4. Applications to Scroll Surfaces. Suppose now that the surface $S_{y}$ is a scroll. Let $v=$ const. be the rulings. It follows therefore that $b=0, a^{\prime} \neq 0$. From (15) and (16) we observe that, if we take

$$
R=\frac{\nu(v)}{a^{\prime}}
$$

where $\nu(v)$ is a function of $v$ alone, the curves corresponding to the developables of the $R$ conjugate and $R$ harmonic congruences coincide. In order to satisfy property (b) of $R$, the function $\nu(v)$ must be transformed according to the formula

$$
\bar{\nu}(v)=\nu(v) / V_{v}^{3} .
$$

If we let $z=y_{u}$, we find readily that the four pairs of functions $(y, z)$ are solutions of differential equations of the form

$$
\begin{aligned}
& y_{v v}+p_{11} y_{v}+p_{12} z_{v}+q_{11} y+q_{12} z=0, \\
& z_{v v}+p_{21} y_{v}+p_{22} z_{v}+q_{21} y+q_{22} z=0
\end{aligned}
$$

wherein

$$
\begin{aligned}
& p_{11}=2 b^{\prime}, p_{12}=0, \quad q_{11}=c^{\prime}, \quad q_{12}=2 a^{\prime}, \\
& p_{21}=2 b_{u}^{\prime}, p_{22}=2 b^{\prime}, q_{21}=c_{u}^{\prime}-2 a^{\prime} c, q_{22}=c^{\prime}+2 a_{u}^{\prime}-4 a a^{\prime} .
\end{aligned}
$$

Denote by $\theta_{4}$ the invariant* whose vanishing implies that the ruled surface has the two branches of its flecnode curve coincident. From (21) we find that $\theta_{4}$ is expressible in terms of the coefficients of system (1) as follows:

(22) $\theta_{4}=64\left[\left(a_{u}^{\prime}-2 a a^{\prime}\right)^{2}-2 a^{\prime}\left(b_{u v}^{\prime}-c_{u}^{\prime}+2 a^{\prime} c+2 b^{\prime} b_{u}^{\prime}\right)\right]$.

If we use the integrability conditions $\dagger$ of system (1), it is easily verified that $\left(\theta_{4}\right)_{u}=0$. Hence $\theta_{4}$ is a function of $v$ alone. Moreover from (6) we find that the transform $\bar{\theta}_{4}$ of $\theta_{4}$ by (4) is defined by the formula

$$
\bar{\theta}_{4}=\theta_{4} / V_{v}^{4} .
$$

* E. J. Wilczynski, Projective Differential Geometry of Curves and Ruled Surfaces, Leipzig, B. G. Teubner, 1906, p. 104.

$\dagger$ Wilczynski, First memoir, p. 245. 
We observe that, if the ruled surface has the two branches of its flecnode curve distinct, we may take the function $\nu(v)$ of (19) to be

$$
\nu(v)=\theta_{4}^{3 / 4} .
$$

The $R$ harmonic line, the scroll directrix of the first kind, and the generator intersect in the point $y_{u}$ for the choice (19) of $R$. The $R$ conjugate line lies in the plane determined by the scroll directrix of the second kind and the generator.

For the choice (19) of $R$, and from (18), we see that the point $y_{u v}$ lies on the osculating quadric for every choice of $\nu(v)$ if and only if

$$
\left(\log a^{\prime}\right)_{u v}=0
$$

that is, if and only if the curved asymptotics* on $S_{y}$ belong tolinear complexes. Moreover the point $y_{u}$ describes a curved asymptotic on $S_{y}$ as $y$ describes the curve $u=$ const.

Michigan State College

* C. T. Sullivan, Properties of ruled surfaces whose asymptotic curves belong to linear comblexes, Transactions of this Society, vol. 15 (1914), p. 190. 